\title{
Percepção de profissionais de saúde sobre utilização de Tecnologias de Informação e Comunicação
}

\author{
Tânia Harumi Uchida \\ Doutoranda do Programa de Pós-Graduação em Odontologia Integrada - UEM \\ Mestra em Odontologia Integrada - UEM \\ Especialista em Saúde Pública - ESPP/ FIOCRUZ \\ Especialista em Gestão em Saúde - UEM \\ Graduação em Odontologia - UEM \\ \taniaharumi@gmail.com \\ Mitsue Fujimaki \\ Docente Associada do Departamento de Odontologia - UEM \\ Docente Permanente do Programa de Pós-Graduação em Odontologia Integrada (Mestrado/Doutorado) - UEM \\ Mestra em Biologia Patologia Buco Dental - FOP-UNICAMP \\ Doutora em Odontologia (Cariologia) - FOP-UNICAMP \\ Graduação em Odontologia - FOB-USP \\ $\triangle$ mfujimaki@gmail.com \\ Josely Emiko Umeda \\ Pós-Doutora em Odontologia Integrada - UEM \\ Doutora em Ciências Biológicas (Microbiologia) - USP \\ Mestra em Ciências Biológicas (Microbiologia) - USP \\ Graduação em Odontologia - UEM \\ $\triangle$ joumeda@gmail.com

\section{Maura Sassahara Higasi} \\ Docente Assistente do Departamento de Medicina Oral e Odontologia Infantil - UEL \\ Mestra em Saúde Coletiva - UEL \\ Especialista em Saúde Coletiva - UEL \\ Especialista em Bioética - UEL \\ Graduação em Odontologia - UEL \\ $\square$ maura.higasi@gmail.com \\ Pablo Guilherme Caldarelli \\ Docente Adjunto do Departamento de Medicina Oral e Odontologia Infantil - UEL \\ Doutorado em Odontologia (Cariologia) - UNICAMP \\ Mestrado em Odontologia em Saúde Coletiva - UNICAMP \\ Especialista em Ativação de Processos de Mudança na Formação Superior de Profissionais de Saúde - FIOCRUZ \\ Especialista em Saúde Coletiva e da Família - UNICAMP \\ Especialista em Gestão em Saúde - UNIFESP \\ Graduação em Odontologia - UEL \\ $\bowtie$ pablocaldarelli@uel.br
}

Recebido em 26 de abril de 2020

Aceito em 3 de junho de 2020

\section{Resumo:}

As Tecnologias de Informação e Comunicação (TICs) são ferramentas necessárias para a qualificação da gestão em saúde. $O$ objetivo do trabalho foi avaliar a aceitação de profissionais de saúde sobre a utilização das TICs nos serviços do Sistema Único de Saúde (SUS) para a construção coletiva e práticas interprofissionais na qualificação da gestão e atenção à saúde. Inicialmente foram capacitados especializandos em saúde pública da Escola de Saúde Pública do Paraná para utilização de ferramentas livres do Google para compartilhamento de documentos, planilhas, agenda e realização de webconferências. Após a capacitação, foram realizadas entrevistas com um roteiro semiestruturado. As transcrições foram realizadas manualmente e posteriormente, analisadas pela análise de conteúdo 
de Bardin, utilizando o software ATLAS.ti 8.0. Os resultados apontaram fatores facilitadores para a utilização das TICs: "aceitação das TICs no serviço" e "reconhecimento das TICs como auxiliar para a prática da EPS", e barreiras como: "desconhecimento sobre as TICs e ferramentas livres do Google" e "falta de entendimento do conceito e da prática da educação permanente em saúde (EPS)". A capacitação oportunizou novos modelos mentais sobre o processo de trabalho e a apropriação tecnológica. Além disso, contribuiu para a reflexão coletiva e o desenvolvimento do senso crítico, que podem auxiliar na prática da EPS, na otimização do trabalho e na consolidação da interprofissionalidade.

Palavras-chave: Educação permanente em saúde, Tecnologia da informação, Pesquisa qualitativa, Pessoal de Saúde.

\title{
Perception of health professionals about the use of Information and Communication Technologies
}

\begin{abstract}
:
Information and Communication Technologies (ICTs) are tools necessary for the qualification of health management. The objective of this work was to evaluate the acceptation of health professionals about the use of ICTs in Unified Health System (SUS) services for the collective construction and interprofessional practices in the qualification of management and health care. Initially, specialization students in public health from the Public Health School of Paraná were trained to use free tools from Google for sharing documents, spreadsheets, schedules and conducting web conferences. After training, interviews were conducted, with a semi-structured script. The transcriptions were performed manually and posteriorly, analyzed by Bardin's content analysis, using the software ATLAS.ti 8.0. The results indicated facilitating factors for the use of ICTs: "Acceptance of ICTs in the service" and "recognition of ICTs as an auxiliary for the practice of the ongoing health education (OHE)", and barriers such as: "lack of knowledge about ICTs and free tools of Google" and "lack of understanding of the concept and practice of OHE". Training has opportunized new mental models on the work process and technological appropriation. Moreover, it contributed to the collective reflection and the development of the critical sense, which can help in the practice of OHE, in the optimization of work and in the consolidation of interprofessionality.
\end{abstract}

Keywords: Continuing education in health, Information technology, Qualitative research, Health Personnel.

\section{Percepción de profesionales de salud sobre utilización de Tecnologías de Información y Comunicación}

\section{Resumen:}

Las Tecnologías de la Información y Comunicación (TICs) son herramientas necesarias para la cualificación de la gestión sanitaria. El objetivo de este trabajo era evaluar la aceptación de los profesionales de la salud sobre el uso de las TICs en los servicios del Sistema Único de Salud (SUS) para la construcción colectiva y las prácticas interprofesionales en la calificación de la gestión y la atención de la salud. Inicialmente, los estudiantes de especialización en salud pública de la Escuela de Salud Pública de Paraná fueron capacitados para utilizar herramientas gratuitas de Google para compartir documentos, hojas de cálculo, horarios y la realización de conferencias web. Después del entrenamiento, se realizaron entrevistas, con un guión semiestructurado. Las transcripciones fueron realizadas manualmente y luego analizadas por el análisis de contenido de Bardin, utilizando el software ATLAS.ti 8.0. Los resultados indicaron factores facilitadores para el uso de las TICs: "aceptación de las TICs en el servicio" y "reconocimiento de las TICs como auxiliar para la práctica de la EPS", y barreras tales como: "falta de conocimiento sobre las TICs" y "reconocimiento de las TICs como auxiliar para la práctica de la educación permanente en salud (EPS)", y barreras tales como: 
"falta de conocimiento sobre las TICs y herramientas libres de Google" y "Falta de comprensión del concepto y la práctica de EPS". La formación ha oportunizado nuevos modelos mentales sobre el proceso de trabajo y la apropiación tecnológica. Además, contribuyó a la reflexión colectiva y al desarrollo del sentido crítico, que puede ayudar en la práctica de las EPS, en la optimización del trabajo y en la consolidación de la interprofesionalidad.

Palabras clave: Educación permanente en salud, Tecnología de la información, Investigación cualitativa, Personal de Salud.

\section{INTRODUÇÃO}

No Brasil, o Sistema Único de Saúde (SUS) surge com a Constituição de 1988 com o intuito de reorganizar o modelo de atenção à saúde (BRASIL, 1988), por meio dos princípios da universalidade, equidade e integralidade (BRASIL, 1990). O sistema de saúde brasileiro enfrenta desafios, relacionados a questões como a organização e a fragmentação das políticas, o insuficiente financiamento, má gerência de recursos, desigualdades em saúde, complexa relação público-privada na prestação de serviços de saúde, fragilidades dos setores de formação e qualificação dos profissionais de saúde (MIRANDA, MENDES, SILVA, 2017; VIACAVA et al., 2018; BARRETO et al., 2019).

$\mathrm{Na}$ tentativa de superar a deficiência na formação dos profissionais de saúde, estratégias têm sido propostas pelo governo federal para a qualificação de profissionais na atenção à saúde e consequentemente, melhoria na prestação de serviços aos usuários (BRASIL, 2004; PERES, SILVA, BARBA, 2016). Em 2004, o Ministério da Saúde instituiu a Política Nacional de Educação Permanente em Saúde (PNEPS), como estratégia do SUS para a formação e o desenvolvimento dos seus profissionais e trabalhadores, buscando articular a integração entre ensino, serviço e comunidade, base para o desenvolvimento de iniciativas qualificadas ao enfrentamento das necessidades e dificuldades do sistema (BRASIL, 2004). A Educação Permanente em Saúde (EPS) é uma proposta ético-político-pedagógica baseada na problematização das práticas, construção coletiva de propostas de intervenção e de consensos para a resolução de problemas do cotidiano do serviço, apresentando-se como uma estratégia necessária para a qualificação dos serviços de saúde e consequente avanço na consolidação do SUS (UCHIDA, 2016).

Para viabilizar as práticas de EPS, as Tecnologias de Informação e Comunicação (TICs) têm sido utilizadas como ferramentas de mudanças do processo de trabalho (FARIAS et al., 2017). Essas tecnologias têm sido incorporadas de maneira crescente na rotina de trabalho 
dos serviços, entretanto, pouco se conhece sobre as barreiras, os fatores facilitadores e a sua utilização visando a construção coletiva no trabalho interprofissional. Um estudo realizado nos serviços de atenção básica no SUS, utilizando sistemas de informação, demonstrou melhorias na qualidade da atenção (SANTOS et al., 2017). Além disso, também foram verificados benefícios na gestão e atenção a partir do uso de TICs para o monitoramento de doenças, do impacto dos serviços, realização de pesquisas, compartilhamento do conhecimento gerado e educação profissional e popular no Rio de Janeiro (PINTO e ROCHA, 2016).

No entanto, o desconhecimento do profissional em utilizar ferramentas eletrônicas mostrou-se como um fator limitador para a utilização das TICs. No estudo realizado por Gava et al. (2016) foi relatado que os profissionais da atenção básica que trabalhavam com um sistema de informação do SUS realizavam os registros com maior dificuldade, em função do uso de novas tecnologias. Por outro lado, quando o registro ocorria de forma manual, estes relataram ser mais fácil. A demora na inserção de dados foi uma das principais queixas dos profissionais de saúde sobre o sistema informatizado, trazendo a necessidade de treinamento e capacitações para ganharem mais agilidade (GAVA et al., 2016). Considerando a importância e o potencial de contribuição das TICs, além das dificuldades inerentes à implementação de novos processos nos serviços, a educação profissional pode e deve trazer abordagens inovadoras.

A Rede Brasileira de Escolas de Saúde Pública (RedEscola), Escola Nacional de Saúde Pública (ENSP), Fundação Oswaldo Cruz (FIOCRUZ) e Escola de Saúde Pública do Paraná (ESPP) tem realizado uma proposta diferenciada para a qualificação em saúde por meio do Curso de Especialização em Saúde Pública. A proposta central é contribuir para novas práticas e organização do trabalho em saúde, fundamentada na PNEPS. Além de qualificar e formar sanitaristas críticos-reflexivos, tem o objetivo de ampliar o olhar para a realidade políticosocial e promover, de maneira dinâmica, mudanças na realidade de saúde (SOUZA e COSTA, 2017). Neste sentido, as TICs são ferramentas que podem favorecer novas abordagens na formação profissional.

Assim, o objetivo deste trabalho foi avaliar a aceitação de profissionais de saúde sobre a utilização das TICs nos serviços no SUS para a construção coletiva e práticas interprofissionais na qualificação da gestão e atenção à saúde. 


\section{METODOLOGIA}

\section{Delineamento da Pesquisa}

Trata-se de um estudo qualitativo e descritivo, com 16 profissionais de saúde do estado do Paraná, matriculados no curso de especialização em Saúde Pública pela ESPP da Secretaria da Saúde do Estado (SESA). Para a pesquisa foram empregadas entrevistas semiestruturadas, audiogravadas in loco, utilizando um roteiro semiestruturado com questões abertas. Buscouse seguir as diretrizes dos critérios consolidados para relatos de pesquisa qualitativa (COREQ) (TONG, SAINSBURY, CRAIG, 2007).

\section{Participantes da Pesquisa}

O critério de amostragem foi por conveniência com o objetivo de explorar diferentes percepções dos profissionais quanto a experiência, idade, formação acadêmica, local de trabalho, tempo de trabalho no serviço público, condutas e princípios em saúde. Foram incluídos na amostra participantes independente do sexo ou idade, que aceitaram participar da pesquisa. A amostra foi constituída por profissionais da área da saúde (cirurgiõesdentistas, enfermeiros, fisioterapeutas, assistentes sociais, fonoaudiólogos, nutricionistas e médicos veterinários), atuantes nas Regionais de Saúde do Paraná ou Unidades Básicas de Saúde, que encontravam-se matriculados no curso de especialização em Saúde Pública pela ESPP.

O estudo foi submetido ao Comitê Permanente de Ética em Pesquisa Envolvendo Seres Humanos - COPEP - Hospital do Trabalhador/SES/PR, seguindo as diretrizes e normas regulamentadoras de pesquisas envolvendo seres humanos (resolução no 466/2012 do Conselho Nacional de Saúde), obtendo o CAAE $n^{\circ}$ 87253018.0.0000.5225. Todos os participantes foram contatados pessoalmente e as entrevistas foram realizadas mediante assinatura do Termo de Consentimento Livre e Esclarecido e de forma presencial (face a face). A participação foi voluntária e os participantes poderiam retirar a sua autorização em qualquer momento da pesquisa. 


\section{Capacitação}

A capacitação ocorreu em um ambiente de sala de aula, no formato expositivo e carga horária de 10 horas, perfazendo uma atividade dinâmica, intercalando momentos de teoria e prática. A atividade foi desenvolvida em dois momentos: no $1^{\circ}$ foi compartilhado com os profissionais o que são as TICs, para que servem e as principais diferenças da educação continuada (EC) da EPS e no $2^{\circ}$ foi explicado como funcionam as ferramentas livres do Google (Gmail, Google Drive, Google Agenda, Google HangOut, Google Talk e Google Formulário). Essas ferramentas livres disponíveis no Google foram escolhidas por serem gratuitas, de fácil acesso e manuseio. Além disso, elas permitem o compartilhamento de documentos, planilhas, agenda e realização de webconferências (GOOGLE, 2008). Cada profissional da saúde utilizou um computador com acesso à internet. A responsável pela capacitação foi uma pesquisadora com experiência em utilização das TICs.

\section{Entrevistas}

Foram realizadas duas entrevistas, antes e depois da capacitação, ambas foram audiogravadas in loco, transcritas e analisadas segundo o método de análise de conteúdo proposto por Bardin (2011), utilizando o software ATLAS.ti 8.

Houve apenas uma entrevistadora responsável que recebeu treinamento prévio sobre as temáticas da pesquisa qualitativa e semiótica da comunicação, desta forma estava familiarizada com os termos utilizados pelos entrevistados e com a suas rotinas de trabalho. Não houve nenhum vínculo de interesse ou de trabalho entre ambas as partes com o objetivo de manter a imparcialidade durante as entrevistas e análise dos dados. Durante abordagem, os entrevistados foram esclarecidos sobre a identificação da entrevistadora, quanto a sua formação e quanto à importância da pesquisa, seus objetivos e como seriam conduzidas as entrevistas.

As entrevistas foram conduzidas por um roteiro com uma duração aproximada de dez a vinte minutos de duração e foram gravadas mediante autorização dos participantes. A saturação das falas foi utilizada como ponto final para a coleta de dados. Foi utilizado um gravador portátil para o registro das entrevistas. 
A transcrição das mesmas foi realizada manualmente pela entrevistadora para possibilitar uma leitura longitudinal que permitiu editá-las, sem alterá-las. A revisão das transcrições foi realizada por um pesquisador com experiência em metodologias qualitativas e com treinamento em transcrições de entrevistas. A confidencialidade dos dados foi mantida, não foi identificada as respostas antes da análise e foram tomados todos os cuidados para não expor detalhes que pudessem identificar os entrevistados.

\section{Roteiros}

A dinâmica da entrevista seguiu um roteiro de perguntas, porém a entrevistadora pôde conduzir o diálogo a fim de obter as informações desejadas sem fugir da proposta ou mesmo desviar do objetivo da pesquisa. Dentro dessa abordagem, procurou dar liberdade para que o entrevistado falasse o que julgasse importante.

Os roteiros utilizados na capacitação foram compostos por tópicos que versavam sobre o conhecimento das TICs e ferramentas livres do Google, utilização das TICs no processo de trabalho e diferenciação de EC de EPS.

\section{Análise dos Dados}

As entrevistas foram realizadas até o ponto de saturação dos dados. Para isso, as falas foram pré-codificadas e pré-analisadas de modo transversal e intuitivo ao longo do estudo pela entrevistadora, o revisor das transcrições e mais um participante do trabalho, possibilitaram assim a identificação do ponto de redundância dos dados. A codificação e análise das entrevistas pela técnica categorial de Bardin e suas transcrições foram realizadas após o término de todas as entrevistas, com auxílio do software The Qualitative Data Analysis \& Research Software 8.0 (Atlas.ti ${ }^{\circledR}$ Scientific Software Development, Berlim, Alemanha). As expressões chave e as ideias centrais, designadas como unidades de registro, foram categorizadas em códigos de acordo com o mesmo sentido, sentido equivalente ou em sentido complementar. O processo de análise dos dados foi realizado por meio da leitura das entrevistas transcritas, à luz do referencial teórico da PNEPS. 


\section{RESULTADOS}

Foram entrevistados neste estudo 16 profissionais da área da saúde, sendo 37,5\% (n= 6) profissionais atuantes nas Regionais de Saúde, 75\% (n=12) profissionais contratados sob o regime estatutário, 37,5\% ( $n=6)$ enfermeiros, $87,5 \%(n=14)$ do sexo feminino, 56,25\% ( $n=9)$ na faixa etária de 30 a 39 anos de idade e 37,5\% (n=6) dos profissionais com mais de 10 anos de trabalho no serviço público (Tabela 1 ).

As principais falas, obtidas das entrevistas, estão apresentadas no Quadro 1, dentro de seus respectivos códigos. 
Tabela 1 - Perfil dos profissionais de saúde do estado do Paraná, matriculados no curso de especialização em Saúde Pública pela ESPP.

Questionamento

Frequência (n)
Porcentagem (\%)

\begin{tabular}{lcc} 
Gênero & & \\
Masculino & 2 & 12,5 \\
Feminino & 14 & 87,5 \\
Total & 16 & 100 \\
\hline
\end{tabular}

\section{Idade}

20-29 anos $\quad 2 \quad 12,5$

30-39 anos $\quad 9 \quad 56,25$

40-49 anos $\quad 4 \quad 25$

Mais de 50 anos $\quad 1 \quad 6,25$

$\begin{array}{lcc}\text { Total } & 16 & 100\end{array}$

\section{Tempo de trabalho no serviço público}

Até 1 ano

6,25

Mais de um ano e menos de 5

31,25

Mais de cinco anos e menos de 10

Mais de 10 anos

37,5

Total

Curso de Graduação

Odontologia

Enfermagem

Nutrição

Medicina Veterinária

Fisioterapia

Fonoaudiologia

Psicologia

\section{Local de Trabalho}

Gestão

Regional de Saúde do estado do Paraná

Unidade Básica de Saúde (UBS)

Núcleo de Apoio à Saúde da Família (NASF)

Consórcio de Saúde

Total

1

6

3

5

1

16
6,25

37,5

18,75

31,25

6,25

100

Forma de Contratação

Estatutário

Consolidação das Leis do Trabalho (CLT)

Fonte: própria. 
Percepção de profissionais de saúde sobre utilização

de Tecnologias de Informação e Comunicação

Quadro 1 - Códigos e respectivas unidades de registro indicando os fatores facilitadores e as barreiras encontradas pelos profissionais de saúde do estado do Paraná matriculados no curso de especialização em Saúde Pública pela ESPP, antes e após a capacitação.

\begin{tabular}{|c|c|c|c|c|c|}
\hline & Fatores Facilitadores & Unidades de Registro & & Barreiras & Unidades de Registro \\
\hline 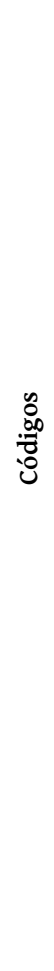 & $\begin{array}{c}\text { Reconhecimento das TICs } \\
\text { como auxiliar para a prática } \\
\text { da EPS }\end{array}$ & $\begin{array}{l}\text { EF10 (após a capacitação): "A TIC é uma forma de por em prática a } \\
\text { EPS. O meu processo de trabalho é todo baseado em dar capacitação, } \\
\text { nós temos que capacitar os municípios sobre os diversos assuntos. } \\
\text { Muitas vezes você capacita uma pessoa de um município e no ano } \\
\text { seguinte esse mesmo profissional não se encontra mais lá, ou porque } \\
\text { mudou de residência, de município, ele não está mais fazendo aquele } \\
\text { mesmo trabalho. A partir das TICs a gente consegue desenvolver, por } \\
\text { exemplo, aulas on line com aqueles assuntos, ao invés de estarmos indo } \\
\text { pessoalmente a gente pode estar disponibilizando para eles aos } \\
\text { materiais e fazer com que as pessoas de alguma forma assistam, vejam } \\
\text { os materiais e depois avaliar se essas pessoas estão mesmo } \\
\text { capacitadas" } \\
\text { EF5 (após a capacitação): "Dentro do meu processo trabalho serviria } \\
\text { para tornar mais efetiva a comunicação entre as Unidades Básicas de } \\
\text { Saúde, secretarias de saúde e hospitais. A TIC seria sim uma forma de } \\
\text { colocar a EPS em prática no serviço de forma efetiva. Por meio de cursos } \\
\text { preparatórios, cursos que a regional envia facilitaria o envio desses } \\
\text { cursos para outros profissionais" } \\
\text { EF12 (após a capacitação): "É uma forma de interligar as ações que } \\
\text { são executadas, por exemplo, no meu trabalho temos que fazer uma } \\
\text { lista das açôes que a gente executa e essa lista deve ser preenchida por } \\
\text { todos os profissionais e pela coordenadora do serviço, cada um faz de } \\
\text { uma forma e depois a coordenadora deve passar tudo para o } \\
\text { computador depois. E, por exemplo, se um documento como este ficasse } \\
\text { online, como foi mostrado na capacitação, isso já facilitaria porque aí } \\
\text { todo mundo mexeria ali no documento e no final do mês o documento } \\
\text { estaria pronto. A TIC com certeza seria uma forma de por a EPS em } \\
\text { prática, por exemplo, por meio de uma capacitação" }\end{array}$ & $\begin{array}{l}0 \\
: 0 \\
: 0 \\
0 \\
0\end{array}$ & $\begin{array}{l}\text { Desconhecimento sobre as } \\
\text { TICs e ferramentas livres do } \\
\text { Google }\end{array}$ & $\begin{array}{l}\text { EI7 (antes da capacitação): "Essas TICs eu nunca ouvi falar" } \\
\text { EI11 (antes da capacitação): "Já ouvi falar só por conceito, mas não } \\
\text { tenho conhecimento" } \\
\text { EI5 (antes da capacitação): "Também não, só sei abrir o e-mail e olhar } \\
\text { o que tem lá e mais nada” }\end{array}$ \\
\hline
\end{tabular}




\begin{tabular}{|c|c|c|c|}
\hline Aceitação das TICs no serviço & $\begin{array}{l}\text { EF1 (após a capacitação): "Eu conseguiria sim inserir a TIC no meu } \\
\text { processo de trabalho. Poderia usar o drive, colocar todos os meus } \\
\text { arquivos, planilhas e aí entrar em contato também com as pessoas do } \\
\text { meu setor ou até mesmo pessoas responsáveis dos municípios" } \\
\text { EF6 (após a capacitação): "A TIC me ajudaria a otimizar o meu } \\
\text { serviço. Já fiquei pensando em várias formas, como compartilhar } \\
\text { documentos. Fiquei pensando em várias coisas que daria para serem } \\
\text { feitas no NASF, tem muitos projetos que nós acabamos redigindo em } \\
\text { comum e aí para otimizar estaríamos compartilhando o mesmo } \\
\text { documento, achei bem legal. E serviria para compartilhar também } \\
\text { documentos, textos, a agenda. No e-mail do Gmail eu já vi essas } \\
\text { ferramentas, mas nunca tive a curiosidade em 'fuçar' o que seriam } \\
\text { aquelas coisas. A única coisa que eu conhecia era o drive, mas só } \\
\text { conhecia a parte de compartilhar documentos, nem imaginava as } \\
\text { outras funçôes. Então eu acho que tudo isso nos auxiliaria em várias } \\
\text { coisas" } \\
\text { EF7 (após a capacitação): "Sim, com certeza, porque a parte mais } \\
\text { difíil de trabalhar na saúde públicaéa sobrecarga de trabalho de cada } \\
\text { profissional e ai muitas vezes você não consegue trocar informações, } \\
\text { conhecimentos entre os membros da própria equipe ou de outros } \\
\text { setores ainda" } \\
\text { EF8 (após a capacitação): "[...] No meu processo trabalho seria uma } \\
\text { forma de eu me ausentar menos no meu trabalho. Me ajudaria a me } \\
\text { manter mais dentro da unidade e sempre buscando por novos } \\
\text { conhecimentos de forma mais dinâmica e atualizada" }\end{array}$ & $\begin{array}{l}\text { Falta de entendimento do } \\
\text { conceito e da prática da EPS }\end{array}$ & $\begin{array}{l}\text { EF10 (após a capacitação): "Para mim é a mesma coisa, não existe } \\
\text { diferença entre a continuada e a permanente" } \\
\text { EF4 (após a capacitação): "Não é totalmente claro para mim, mas } \\
\text { acendeu uma luz no fim do túnel. Não sei ainda descrever uma diferença } \\
\text { entre educação continuada e educação permanente em saúde" } \\
\text { EF5 (após a capacitação): "Me confundo ainda quanto as diferenças } \\
\text { entre uma e outra. Eu sei que uma é coletiva e outra é individual, não sei } \\
\text { ao certo qual é qual" }\end{array}$ \\
\hline
\end{tabular}

Fonte: própria. 
Em relação às ferramentas livres do Google, 50\% $(n=8)$ dos profissionais entrevistados elencaram o Google Drive como a plataforma que teria maior utilidade na prática do serviço, seguidos do Google Agenda e Google Formulário (Gráfico 1).

Gráfico 1 - Distribuição percentual das ferramentas livres do Google que mais auxiliariam a prática do serviço, segundo os profissionais da saúde do estado do Paraná matriculados no curso de especialização em Saúde Pública pela ESPP.

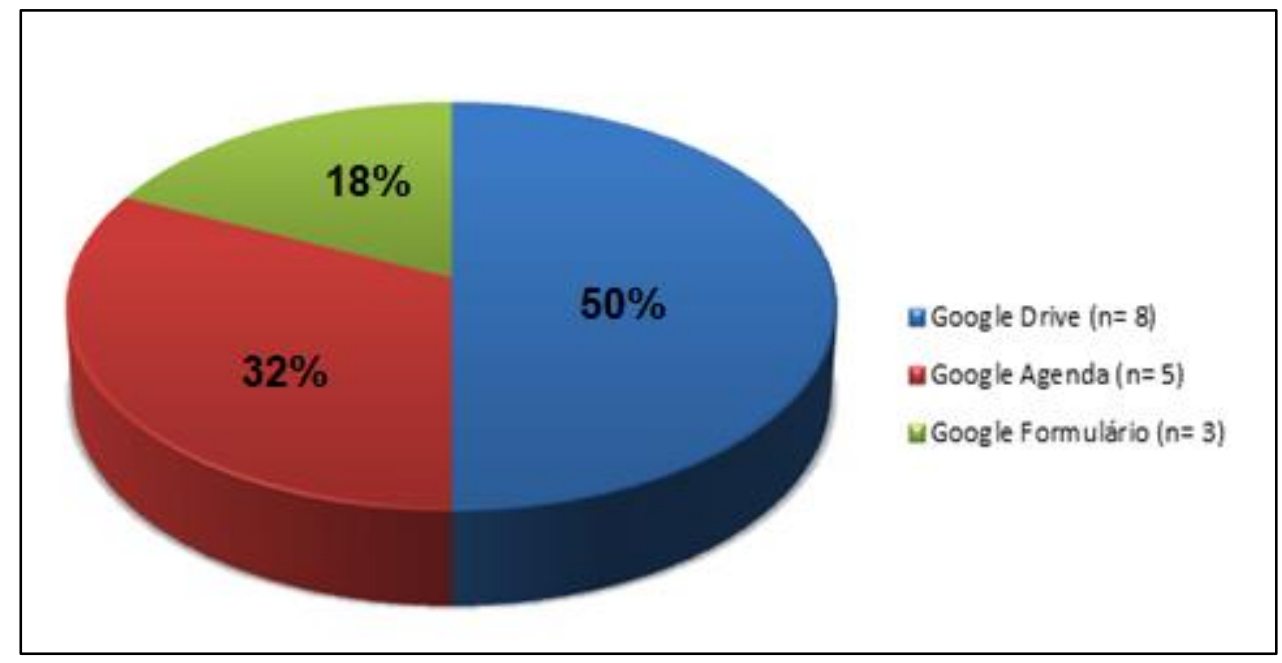

Fonte: Própria.

Os resultados apontaram fatores facilitadores para a utilização das TICs: "aceitação das TICs no serviço" e "reconhecimento das TICs como auxiliar para a prática da EPS". Quanto às barreiras emergiram códigos: "desconhecimento sobre as TICs e ferramentas livres do Google" e "falta de entendimento do conceito e da prática da EPS".

\section{DISCUSSÃO}

Este estudo avaliou a aceitação de profissionais de saúde do estado do Paraná, matriculados no curso de especialização em Saúde Pública pela ESPP da SESA, sobre a 
utilização das TICs para a prática da EPS. Nas entrevistas iniciais, antes da capacitação, os profissionais mostraram ter conhecimento superficial sobre as TICs e ferramentas livres do Google, além disso, houve a percepção de que as TICs seriam ferramentas a serem utilizadas na otimização e qualificação da gestão dos serviços de saúde. Após a capacitação, foi aprimorado o conhecimento sobre as TICs e sua aplicabilidade no processo de trabalho e para a prática da EPS, podendo constatar a dificuldade de pôr em prática a EPS no serviço e o desconhecimento da diferença entre EC e EPS.

Dentre os fatores facilitadores que emergiram na pesquisa foi possível verificar as TICs como uma ferramenta que pode otimizar processo de trabalho. As percepções dos participantes da pesquisa (antes da capacitação) mostraram que as TICs são ferramentas disponíveis na internet e que melhoraram o processo de trabalho, otimizando tempo e auxiliando no planejamento e organização das ações em saúde e no cuidado dos pacientes. Após a capacitação, os profissionais sentiram-se aptos para implementar a TIC no serviço, emergindo o código “aceitação das TICs no serviço”. Nas falas dos entrevistados ficou evidente que a utilização das tecnologias seria para melhoria do serviço e o Google drive seria a plataforma que mais utilizariam por terem a possibilidade de compartilhar documentos, projetos, relatórios, propiciando trocas de informações e conhecimentos entre membros da equipe (Gráfico 1).

Esse resultado vem de encontro com os estudos qualitativos realizados por Santos et al. (2017) e Pinto e Rocha (2016) que ao descreverem a incorporação de TICs na atenção básica, apontaram que essas tecnologias contribuíram para a melhoria da qualidade do serviço por otimizar o tempo, facilitar a comunicação entre a equipe e ampliar a capacidade de gestão das políticas de saúde.

No entanto, para que haja a incorporação das TICs no processo de trabalho é importante considerar fatores como: qualidade da interface, usabilidade, funcionalidade dos recursos, qualidade dos dados e integração com sistemas externos e o domínio das tecnologias pelo trabalhador na inclusão dessas tecnologias na saúde (SANTOS et al., 2017). No presente estudo, ficou nítido nos participantes o entendimento superficial sobre as TICs e das ferramentas livres do Google. Quando foi questionado aos entrevistados sobre o conceito das TICs e ferramentas livres do Google, alguns relataram possuírem um conhecimento superficial 
sobre os temas, relacionando as TICs com a internet, enquanto outros expuseram nenhum tipo de familiaridade com os temas, não sabendo o que são as TICs e tendo o Gmail apenas como uma plataforma de correspondência eletrônica. Dentro desse contexto, o "desconhecimento sobre as TICs e ferramentas livres do Google" foi um código que emergiu como uma barreira nas falas dos entrevistados, corroborando com os estudos de Cavalcante et al. (2016), Pinto et al. (2014) e Garcia e Baptista (2007), que evidenciaram a dificuldade da utilização das TICs por parte dos profissionais. Essa dificuldade estava relacionada principalmente à sua falta de habilidade para operar essas tecnologias (FARIAS et al., 2017). No trabalho de Silva et al. (2015) foi observado que, em países em desenvolvimento, os profissionais de saúde enfrentam grandes desafios socioeconômicos ao mesmo tempo em que vivenciam as transformações relacionadas às tecnologias.

Ao analisar o código "desconhecimento sobre as TICs e ferramentas livres do Google" na perspectiva da EPS foi possível verificar que por mais que as dificuldades de implementação das TICs nos serviços existam, é irrefutável mencionar a importância delas ao disponibilizar uma ampla gama de aplicações para facilitar a execução e controle dos serviços públicos e da gestão. Sendo, então, as TICs consideradas como importantes mediadoras para a prática da EPS, melhorando a tomada de decisão administrativa, a prestação de serviços aos usuários e outras mudanças organizacionais do governo local, necessárias a um melhor desempenho das atividades do setor público (MADEIRA, GUIMARÃES, MENDES, 2017).

Em contrapartida, nas falas dos entrevistados foi possível identificar diferentes percepções quanto à importância do "reconhecimento das TICs como auxiliar para a prática da EPS", como: a melhora da comunicação entre profissionais da saúde e gestores das Unidades Básicas de Saúde e Regionais de Saúde, a facilitação da comunicação multi e interprofissional e a garantia da qualidade da assistência prestada à população. Em um estudo realizado com enfermeiros da atenção básica foi destacada a relevância da consolidação da EPS para o processo de trabalho e a importância da EPS na qualidade da assistência, mesmo diante de várias dificuldades (SALUM e PRADO, 2014). Em outro estudo, a educação permanente foi capaz de promover mudanças no processo de trabalho, de viabilizar formação crítica e reflexiva dos profissionais de saúde, de fortalecer a participação social e de aproximar a gestão das questões locais de saúde (VIANA et al., 2015). 
Sob a perspectiva da PNEPS, é importante que o profissional de saúde desenvolva uma postura crítico-reflexivo e, para isso, é necessária a aquisição de conhecimentos e competências técnicas e relacionais, de forma a promover o desenvolvimento profissional e pessoal dos sujeitos. Na proposta da educação permanente, para a capacitação das equipes, os conteúdos dos cursos e as tecnologias a serem utilizadas devem ser determinados, a partir da observação dos problemas que ocorrem no cotidiano do trabalho e que precisam ser solucionados para que os serviços prestados ganhem qualidade e os usuários fiquem satisfeitos com a atenção prestada. Torna-se cada vez mais evidente a necessidade de educação no ambiente dos trabalhadores da área da saúde, pois o acelerado crescimento dos espaços de trabalho tem demandado uma atuação profissional pautada no conhecimento e no desenvolvimento de competências e habilidades para tomada de decisões (SILVA et al., 2015).

No presente trabalho, pôde-se observar que não foi compreendido entre os profissionais de saúde a diferença entre EC e EPS. Foi possível analisar nas falas uma "falta de entendimento do conceito e da prática da EPS", enquanto alguns entrevistados acreditavam que EC e EPS seriam sinônimos, outros faltava clareza no entendimento entre uma e outra. No estudo de revisão integrativa realizada por Campos et al. (2017), também foi possível verificar a dificuldade dos profissionais da saúde diferenciar EC de EPS. $O$ estudo demonstrou que ainda há confusão ou desconhecimento da definição de educação permanente por profissionais e gestores, sendo muitas vezes utilizada como sinônimo de "educação continuada", dificultando o processo de mudança na prática.

As TICs deram um novo direcionamento à forma de comunicação em todas as áreas do conhecimento. A tecnologia passou a desempenhar um papel importante na estrutura organizacional da sociedade permitindo o processamento, o armazenamento, a difusão da informação e do conhecimento (PINOCHET, LOPES, SILVA, 2017). No âmbito da saúde, as TICs têm apoiado a organização e gestão dos processos institucionais, integrando os recursos computacionais à prática profissional (GONÇALVES et al., 2013; LORENZETTI et al., 2012). Além disso, destaca-se o importante papel desempenhado no auxílio aos profissionais para adquirirem competências necessárias para tomarem decisões, solucionarem problemas e contribuírem para a melhoria da saúde dos usuários (ONU, 2009). 


\section{CONCLUSÃO}

A capacitação dos profissionais de saúde oportunizou novos modelos mentais sobre o processo de trabalho e a apropriação tecnológica. Essas tecnologias eram desconhecidas pela maioria dos profissionais, mas todos estavam abertos ao aprendizado de novas funcionalidades, tendo sido percebido o potencial de contribuição e otimização do tempo e de recursos com ferramentas livres da internet.

Além disso, a experiência da capacitação do uso de TICs no Curso de Especialização em Saúde Pública pode ter auxiliado na melhor compreensão da prática da EPS no dia a dia do serviço e a construção coletiva, demonstrados no uso de documentos com edição coletiva, agendas compartilhadas e construção de formulários eletrônicos para redação de relatórios de equipes. A integração e interação em ambiente virtual gerada pelas TICs podem estimular o diálogo e a prática da interprofissionalidade em ambiente real.

Dessa forma, é relevante que mais trabalhos sejam desenvolvidos no intuito de avaliar se a incorporação das TICs tem favorecido a melhoria da gestão e da atenção, auxiliando a prática da EPS nos serviços no SUS. Vale ressaltar que esta incorporação pode ocorrer em velocidades diferentes para cada indivíduo, bem como para o serviço, havendo a necessidade de preparo, treinamento, acompanhamento e avaliação das etapas necessárias ao longo do tempo. Um fator limitador deste trabalho foi a ausência de profissionais da medicina entre os participantes da pesquisa.

Por fim, conclui-se que a aceitação dos profissionais na utilização das TICs para a construção coletiva interprofissional foi positiva, demonstrando ser uma estratégia viável para a melhoria dos processos de trabalho e qualificação da gestão e atenção nos serviços de saúde no SUS. 


\section{REFERÊNCIAS}

BARRETO, R.M.A.; ALBUQUERQUE, I.M.N.; CARNEIRO, M.S.M.; RIBEIRO, M.A. Contribuição da avaliação para qualificação da Atenção Primária à Saúde: revisão integrativa da literatura. Revista Saúde (Sta. Maria), Santa Maria, v. 45, n. 3, p. 1-16, 2019. Disponível em: https://periodicos.ufsm.br/revistasaude/article/view/35027/pdf. Acesso em: 20 de fevereiro de 2020.

BARDIN, L. Análise de Conteúdo. 7. ed. São Paulo: Edições, 2011.

BRASIL. Constituição (1988). Constituição da República Federativa do Brasil. 5. ed. atual. e ampl. São Paulo: Atlas, $2005 . \quad$ Disponível em: https://www.senado.leg.br/atividade/const/con1988/CON1988_05.10.1988/CON1988.asp. Acesso em: 18 de novembro de 2018.

BRASIL. Lei n. 8.080, de 19 de setembro de 1990. Dispõe sobre as condições para a promoção, proteção e recuperação da saúde, a organização e o funcionamento dos serviços correspondentes, e da outras providências. Diário Oficial da União, Poder Executivo, Brasília, DF, 20 set. 1990. p. 18055. Disponível em: http://www.planalto.gov.br/ccivil_03/leis/18080.htm. Acesso em: 20 de novembro de 2018.

BRASIL. Ministério da Saúde. Portaria GM/MS n. 198, de 13 de fevereiro de 2004. Institui a Política Nacional de Educação Permanente em Saúde. Brasília: Ministério da Saúde, 2004. Disponível em: http://bvsms.saude.gov.br/bvs/saudelegis/gm/2017/MatrizesConsolidacao/comum/13150.html. Acesso em: 20 de novembro de 2018.

CAMPOS, K.F.C.; SENA, R.R.; SILVA, K.L. Educação permanente nos serviços de saúde. Escola Anna Nery, Rio de Janeiro, v. 21, n. 4, p. 01-10, 2017. Disponível em: http://www.scielo.br/scielo.php?pid=S1414-

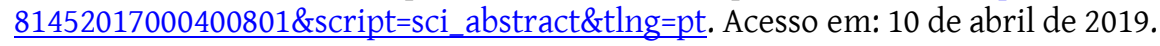

CAVALCANTE, R.B.; GONTIJO, T.L.; SILVA, L.T.C.; ESTEVES, C.J.S.; DINIZ, F.A.; VASCONCELOS, D.D. Experiências de enfermeiros na educação a distância: um olhar sobre as dimensões interação e autonomia. Cogitare Enfermagem, Curitiba, v. 21, n. 2, p. 01-09, 2016. Disponível em: https://revistas.ufpr.br/cogitare/article/view/44756/. Acesso em: 24 de fevereiro de 2019.

FARIAS, Q.L.T.; ROCHA, S.P.; CAVALCANTE, A.S.P.; DINIZ, J.L.; NETO PONTE, O.A.; VASCONCELOS, M.I.O. Implicações das tecnologias de informação e comunicação no processo de educação permanente em saúde. Revista Eletrônica de Comunicação, Informação e Inovação em Saúde, Rio de Janeiro, v. 11, n. 4, p. 1-11, 2017. Disponível em: https://www.reciis.icict.fiocruz.br/index.php/reciis/article/view/1261. Acesso em: 02 de fevereiro de 2019.

GARCIA, R.M.; BAPTISTA, R. Educação a distancia para a qualificação dos profissionais do SUS: perspectivas e desafios. Revista Baiana de Saúde Pública, Salvador, v. 31, n. 1, p. 70-8, 2007. Disponível em: http://bases.bireme.br/cgi-

bin/wxislind.exe/iah/online/?IsisScript=iah/iah.xis\&src=google\&base=LILACS\&lang=p\&nextAction=lnk\&expr Search=478485\&indexSearch=ID. Acesso em: 15 de fevereiro de 2019.

GAVA, M.; FERREIRA, L.S.; PALHARES, D.; MOTA, E.L.A. Incorporação da tecnologia da informação na Atenção Básica do SUS no Nordeste do Brasil: expectativas e experiências. Revista Ciência \& Saúde Coletiva, Rio de Janeiro, v. 21, n. 3, p. 891-902. Disponível em: https://www.scielosp.org/article/csc/2016.v21n3/891-902/. Acesso em: 25 de fevereiro de 2020.

GONÇALVES, J.P.P.; BATISTA, L.R.; CARVALHO, L.M.; OLIVEIRA, M.P.; MOREIRA, K.S.; LEITE, M.T.S. Prontuário Eletrônico: uma ferramenta que pode contribuir para a integração das Redes de Atenção à Saúde. Saúde em Debate, Rio de Janeiro, v. 37, n. 96, p. 43-50, 2013. Disponível em: http://www.scielo.br/scielo.php?script=sci_arttext\&pid=S0103-11042013000100006. Acesso em: 11 de fevereiro de 2019. 
GOOGLE. O uso da internet no Brasil para pesquisas sobre saúde, doenças e medicamentos. Media Screen, 2008. Acesso em: 20 de janeiro de 2020.

LORENZETTI, J.; TRINDADE, L.L.; PIRES, D.E.P.; RAMOS, F.R.S. Tecnologia, inovação tecnológica e saúde: uma reflexão necessária. Texto \& Contexto Enfermagem, Florianópolis, v. 21, n. 2, p. 432-9, 2012. Disponível em: http://www.scielo.br/scielo.php?script=sci_arttext\&pid=S0104-07072012000200023. Acesso em: 05 de fevereiro de 2019.

MADEIRA, G.S.; GUIMARÃES, T.; MENDES, L.S. Construindo governança eletrônica de cidades. Um modelo de implementação de soluções para inovação e otimização da gestão pública. Revista de Gestão dos Países de Língua Portuguesa, Lisboa, v. 16, n.2, p. 55-71, 2017. Disponível em: http://www.scielo.mec.pt/scielo.php?script=sci_arttext\&pid=S1645-44642017000200005. Acesso em: 04 de fevereiro de 2019.

MIRANDA, G.M.D.; MENDES, A.C.G.; SILVA, A.L.A. O desafio da organização do Sistema Único de Saúde universal e resolutivo no pacto federativo brasileiro. Revista Saúde \& Sociedade, São Paulo, v. 26, n. 2, p. 329-335, 2017. Disponível em: https://www.scielosp.org/pdf/sausoc/2017.v26n2/329-335/pt. Acesso em: 25 de fevereiro de 2020 .

ONU. Organização das Nações Unidas para a Educação, a Ciência e a Cultura. Padrões de competências em TIC para professores: diretrizes de implementação. Paris: UNESCO, 2009. Disponível em: https://unesdoc.unesco.org/ark:/48223/pf0000156209_por. Acesso em: 01 de fevereiro de 2019.

PERES, C.; SILVA, R.F.; BARBA, P.C.S.D. Desafios e potencialidades do processo de educação permanente em saúde. Revista Trabalho, Educação e Saúde, Rio de Janeiro, v. 14, n. 3, p. 783-801,set./dez. 2016. Disponível em: http://www.scielo.br/pdf/tes/v14n3/1981-7746-tes-1981-7746-sol00016.pdf. Acesso em: 25 de fevereiro de 2020 .

PINOCHET, L.H.C.; LOPES, A.S.; SILVA, J.S. Inovações e tendências aplicadas nas tecnologias de informação e comunicação na gestão da saúde. Revista de Gestão em Sistemas de Saúde, São Paulo, v. 3, n. 2, p. 11-19, 2014. Disponível em: http://www.revistargss.org.br/ojs/index.php/rgss/article/view/88. Acesso em: 24 de fevereiro de 2019.

PINTO, H.A.; FERLA, A.A.; CECCIM, R.B.; FLORÊNCIO, A.R.; BARBOSA, M.G.; STÉDILE, N.L.R.; ZORTEA, A.P.; MATOS, I.B. Atenção Básica e Educação Permanente em Saúde: cenário apontado pelo Programa Nacional de Melhoria do Acesso e da Qualidade da Atenção Básica (PMAQ-AB). Divulgação em Saúde para Debate, Rio de Janeiro, n. 51, p. 145-160, 2014. Disponível em: http://pesquisa.bvsalud.org/portal/resource/pt/lil-771505. Acesso em: 21 de fevereiro de 2019.

PINTO, L.F.; ROCHA, C.M.F. Inovações na Atenção Primária em Saúde: o uso de ferramentas de tecnologia de comunicação e informação para apoio à gestão local. Revista Ciência \& Saúde Coletiva, Rio de Janeiro, v. 21, n. 5, p. 1433-1448, 2016. Disponível em: https://www.scielosp.org/article/csc/2016.v21n5/1433-1448/. Acesso em: 25 de fevereiro de 2020.

SALUM, N.C.; PRADO, M.L. Continuing education in the development of competences in nurses. Texto \& Contexto Enfermagem, Florianópolis, v. 23, n. 2, p. 301-18, 2014 . Disponível em: http://www.scielo.br/scielo.php?script=sci_arttext\&pid=S0104-07072014000200301. Acesso em: 20 de fevereiro de 2019.

SANTOS, A.F.; SOBRINHO FONSECA, D.; ARAUJO, L.L.; PROCÓPIO, C.S.D.; LOPES, E.A.S.; LIMA, A.M.L.D.; REIS, C.M.R.; ABREU, D.M.X.; JORGE, A.O.; MATTA-MACHADO, A.T. Incorporação de Tecnologias de Informação e Comunicação e qualidade na atenção básica em saúde no Brasil. Cadernos de Saúde Pública, Rio de Janeiro, v. 33, n. 5, p. 01-14, 2017. Disponível em: http://www.scielo.br/scielo.php?pid=S0102311X2017000505003\&script=sci_abstract\&tlng=pt. Acesso em: 14 de fevereiro de 2019. 
SILVA, J.R.; MEDEIROS, F.B.; MOURA, F.M.S.; BESSA, W.S.; BEZERRA, E.L.M. Uso das Tecnologias de Informação e Comunicação no Curso de Medicina da UFRN. Revista Brasileira de Educação Médica, Brasília, v. 39, n. 4, p. 537-541, 2015. Disponível em: http://www.scielo.br/scielo.php?pid=S010055022015000400537\&script=sci_abstract\&tlng=pt. Acesso em: 10 de março de 2019.

SILVA, D.S.J.R.; DUARTE, L.R. Educação Permanente em Saúde. Revista da Faculdade de Ciências Médicas de Sorocaba, Sorocaba, v. 17, n. 2, p. 104-105, 2015. Disponível em: https://revistas.pucsp.br/index.php/RFCMS/article/view/23470. Acesso em: 01 de fevereiro de 2019.

SOUZA, R.M.P.; COSTA, P.P. Redescola e a nova formação em Saúde Pública. Rio de Janeiro: ENSP/ REDESCOLA, 2017. Disponível em: http://rededeescolas.ensp.fiocruz.br/redescola-e-nova-formacao-em-saude-publica. Acesso em: 19 de setembro de 2018.

TONG, A.; SAINSBURY, P.; CRAIG, J. Consolidated criteria for reporting qualitative research (COREQ): a 32-item checklist for interviews and focus group. International Journal for Quality in Health Care, v. 19. n. 6, p. 349357, 2007. Disponível em: https://academic.oup.com/intqhc/article/19/6/349/1791966. Acesso em: 19 de setembro de 2018 .

UCHIDA, T.H. Revisão Sistemática e Metassumarização sobre condutas para qualificação da gestão em Saúde Bucal no Brasil. Dissertação (Mestrado em Odontologia Integrada) - Departamento de Odontologia, Universidade Estadual de Maringá, UEM, Maringá, 2016. Disponível em: http://www.pgo.uem.br/dissertacoes/dissertacoes-dos-egressos-de-2016-1/TniaHarumiUchida.pdf. Acesso em: 20 de novembro de 2018.

VIACAVA, F.; OLIVEIRA, R.A.D.; CARVALHO, C.C.; LAGUARDIA, J.; BELLIDO, J.G. SUS: oferta, acesso e utilização de serviços de saúde nos últimos 30 anos. Revista Ciência \& Saúde Coletiva, Rio de Janeiro, v. 23, n. 6, p. 17511762. Disponível em: https://www.scielosp.org/article/csc/2018.v23n6/1751-1762/. Acesso em: 25 de fevereiro de 2020.

VIANA, D.M.S.; NOGUEIRA, C.A.; ARAÚJO, R.S.; VIEIRA, R.M.; RENNÓ, H.M.S.; OLIVEIRA, V.C. A educação permanente em saúde na perspectiva do enfermeiro na estratégia de saúde da família. Revista de Enfermagem do Centro-Oeste Mineiro, São João Del-Rei, v. 5, n. 2, p. 1658-68, 2015. Disponível em: http://www.seer.ufsj.edu.br/index.php/recom/article/view/470. Acesso em: 04 de fevereiro de 2019.

(cc) $\mathrm{Br}$

Este trabalho está licenciado com uma Licença Creative Commons - Atribuição 4.0 Internacional. 